\title{
$\gamma$-ray assisted synthesis of Ni3Se2 nanoparticles stabilized by natural polymer
}

\begin{abstract}
Nickel selenide nanoparticles were synthesized using J-ray irradiation in the presence of natural polymer, chitosan as capping agent. Chitosan is the deacetylated product of chitin, the second most abundant organic resources after cellulose. The nanoparticles were produced using nickel acetate and selenium dioxide and the as-prepared chitosan stabilized nanoparticles were soluble and stable in aqueous solution. The morphology and structure of the nickel selenide nanoparticles were characterized using transmission electron microscope (TEM) and X-ray diffraction (XRD). Optical properties of the nanoparticles were characterized by UVï Visible spectrophotometer and photoluminescent spectroscopy. The XRD result shows that the nickel selenide conformed to Ni3Se2 with crystal structure of rhombohedral. The absorption spectrum of the Ni3Se2 nanoparticles covered from around $300 i ̈ 600 \mathrm{~nm}$ which makes it a potential photovoltaic and optoelectronic device material. In this report, J-ray irradiation provided a ñgreenò, simple and clean route for the synthesis of chitosan stabilized Ni3Se2 nanoparticles. The size and size distribution of the nickel selenide nanoparticles were influenced by the concentration of chitosan and absorbed dose of J-ray irradiation.
\end{abstract}

Keyword: Nickel selenides; J-ray irradiation; Electron microscope; X-ray diffraction; Nanoparticles; Chitosan 